TOKYO J. MATH

VOL. 28, No. 1, 2005

\title{
A Comparison Theorem on Crescents for Kähler Magnetic Fields
}

\author{
Toshiaki ADACHI
}

\author{
Nagoya Institute of Technology \\ (Communicated by Y. Ohnita)
}

\begin{abstract}
For a non-trivial Kähler magnetic field on a Kähler manifold, we consider bow-shapes as substitutions for triangles on a Riemannian manifold. We give a comparison theorem for bow-shapes on a manifold whose sectional curvature is bounded from above.
\end{abstract}

\section{Introduction}

In papers [1, 2, 3] the author studied Kähler manifolds from Riemannian geometric point of view by using Kähler magnetic fields. On a Kähler manifold $(M, J,\langle\rangle$,$) a closed 2-form$ $\mathbf{B}_{\kappa}=\kappa \mathbf{B}_{J}$ which is a constant multiple of the Kähler form $\mathbf{B}_{J}$ on $M$ is said to be a Kähler magnetic field. A smooth curve $\gamma$ parameterized by its arc-length is called a trajectory for $\mathbf{B}_{\kappa}$ if it satisfies the equation $\nabla_{\dot{\gamma}} \dot{\gamma}=\kappa J \dot{\gamma}$. As it is a geodesic when $\kappa=0$, the author would like to investigate Kähler manifolds by use of some properties of trajectories for Kähler magnetic fields.

In Riemannian geometry it is a basic idea to compare the geometry of an arbitrary Riemannian manifold with geometry of a space of constant curvature. Powerful results were first obtained by Rauch, Alexandrov, Toponogov and Bishop, and active development was done by many geometers. In [2] the author studied a comparison theorem on Kähler magnetic Jacobi fields, which was generalized by N. Gouda[8] for general magnetic fields. He gave interesting results on geometry of general manifolds with uniform magnetic fields in $[8,9]$. In this paper, in order to give another light on the study of non-trivial Kähler magnetic fields on general Kähler manifolds, we consider "bow-shapes" which are consisted of trajectories and a kind of geodesics and study a theorem of comparison type. Through out of this paper we suppose $\kappa \neq 0$.

The author is grateful to the referee who read this paper carefully and gave him significant comments.

Received December 18, 2003; revised December 25, 2004

2000 Mathematics subject classification: Primary 53C20, 53C22, 53C55.

The author is partially supported by Grant-in-Aid for Scientific Research (C) (No. 14540075), Ministry of Education, Science, Sports, Culture and Technology. 


\section{Jacobi fields associated with a trajectory}

Let $\gamma$ be a trajectory for a non-trivial Kähler magnetic field $\mathbf{B}_{\kappa}$ on a complete Kähler manifold $M$. We shall call a map $\alpha: \mathbf{R}^{2} \rightarrow M$ a variation of geodesics associated with $\gamma$ if it satisfies the following conditions;

i) $\gamma(s)=\alpha(s, 0)$,

ii) for each $s$ the map $\sigma_{s}(\cdot)=\alpha(s, \cdot)$ is a geodesic,

iii) $\frac{\partial \alpha}{\partial t}(s, 0)$ is parallel to $J \dot{\gamma}(s)$ and satisfies $\kappa\left\langle\frac{\partial \alpha}{\partial t}(s, 0), J \dot{\gamma}(s)\right\rangle>0$.

LEMMA 1. For a variation $\alpha$ of geodesics associated with a trajectory $\gamma$ for $\mathbf{B}_{\kappa}$, we consider a Jacobi field $Y=\frac{\partial \alpha}{\partial s}\left(s_{0}, \cdot\right)$ along a geodesic $\sigma=\alpha\left(s_{0}, \cdot\right)$. Then it satisfies

1) $Y(0)=\dot{\gamma}\left(s_{0}\right)$,

2) $\left.\frac{1}{2} \frac{d}{d t}\|Y(t)\|^{2}\right|_{t=0}=\left\langle\nabla_{\sigma^{\prime}} Y(0), \dot{\gamma}\left(s_{0}\right)\right\rangle=-\kappa\left\langle\sigma^{\prime}(0), J \dot{\gamma}\left(s_{0}\right)\right\rangle<0$,

3) $\nabla_{\sigma^{\prime}} Y(0)$ is contained in the complex vector subspace spanned by $\dot{\gamma}\left(s_{0}\right)$.

If $\alpha$ is a variation of normal geodesics, that is $\left\|\frac{\partial \alpha}{\partial t}(s, t)\right\|=1$ for every $s$, then $Y$ also satisfies $\left\langle\nabla_{\sigma^{\prime}} Y(0), J \dot{\gamma}\left(s_{0}\right)\right\rangle=0$.

Proof. Since $\frac{\partial \alpha}{\partial t}(s, 0)$ is parallel to $J \dot{\gamma}(s)$, we see

$$
0=\frac{d}{d s}\left\langle\frac{\partial \alpha}{\partial t}(s, 0), \dot{\gamma}(s)\right\rangle=\left\langle\frac{\partial}{\partial s}\left(\frac{\partial \alpha}{\partial t}\right)(s, 0), \dot{\gamma}(s)\right\rangle+\left\langle\frac{\partial \alpha}{\partial t}(s, 0), \kappa J \dot{\gamma}(s)\right\rangle,
$$

which shows the second assertion.

If a (local) vector field $V$ along $\gamma$ is orthogonal to both $\dot{\gamma}(s)$ and $J \dot{\gamma}(s)$ at each $s$, we find $\nabla_{\dot{\gamma}} V$ is also orthogonal to both $\dot{\gamma}(s)$ and $J \dot{\gamma}(s)$ by differentiating both sides of the equalities $\langle V(s), \dot{\gamma}(s)\rangle=0$ and $\langle V(s), J \dot{\gamma}(s)\rangle=0$. Differentiating both sides of $\left\langle\frac{\partial \alpha}{\partial t}(s, 0), V(s)\right\rangle=0$, we see

$$
\left\langle\nabla_{\dot{\gamma}} \frac{\partial \alpha}{\partial t}(s, 0), V(s)\right\rangle=-\left\langle\frac{\partial \alpha}{\partial t}(s, 0), \nabla_{\dot{\gamma}} V(s)\right\rangle=0,
$$

and obtain the third assertion.

We find the last assertion by differentiating both sides of $\left\|\frac{\partial \alpha}{\partial t}(s, t)\right\|^{2}=1$ by $s$.

Following Lemma 1, we shall say that a Jacobi field $Y$ along a geodesic $\sigma$ is associated with a trajectory for $\mathbf{B}_{\kappa}$ if it satisfies

i) $Y(0)=-\operatorname{sgn}(\kappa) J \sigma^{\prime}(0) /\left\|\sigma^{\prime}(0)\right\|$,

ii) $\nabla_{\sigma^{\prime}} Y(0)$ is contained in the complex vector subspace spanned by $\sigma^{\prime}(0)$ and satisfies $\left\langle\nabla_{\sigma^{\prime}} Y(0), J \sigma^{\prime}(0)\right\rangle=\kappa\left\|\sigma^{\prime}(0)\right\|^{2}$.

Here $\operatorname{sgn}(a)$ is the signature of a real number $a$. It is clear that every Jacobi field associated with a trajectory for $\mathbf{B}_{\kappa}$ can be obtained by some variation of geodesics associated with this trajectory. When $\sigma$ is a normal geodesic, that is a geodesic of unit speed, we find a Jacobi field $Y$ associated with a trajectory for $\mathbf{B}_{\kappa}$ is of the form $Y=a t \dot{\sigma}(t)-\operatorname{sgn}(\kappa) g(t) J \dot{\sigma}(t)+Y^{\perp}$ with a constant $a$, a function $g$ and a vector field $Y^{\perp}$ along $\sigma$ which is orthogonal to both 
$\dot{\sigma}(t)$ and $J \dot{\sigma}(t)$ at every $t$. The function $g$ and the vector field $Y^{\perp}$ satisfy $g(0)=1, g^{\prime}(0)=$ $-|\kappa|, Y^{\perp}(0)=0, \nabla_{\dot{\sigma}} Y^{\perp}(0)=0$.

LEMMA 2. We consider a surface formed by a variation $\alpha$ of geodesics associated with a trajectory for $\mathbf{B}_{\kappa}$, which may have singularities. This surface is a complex line if and only if the vector $R\left(J \frac{\partial \alpha}{\partial t}, \frac{\partial \alpha}{\partial t}\right) \frac{\partial \alpha}{\partial t}$ is parallel to $J \frac{\partial \alpha}{\partial t}$ at each point. In this case it is totally geodesic.

PROOF. The variation $\alpha$ forms a complex line if and only if the corresponding Jacobi field $Y$ satisfies $Y^{\perp} \equiv 0$. Such case occurs if and only if $R\left(J \frac{\partial \alpha}{\partial t}, \frac{\partial \alpha}{\partial t}\right) \frac{\partial \alpha}{\partial t}$ is parallel to $J \frac{\partial \alpha}{\partial t}$. We show $\alpha$ forms a totally geodesic surface in this case. For the sake of simplicity, we may only treat the case that $\alpha$ is a variation of normal geodesics. In this case we have $\frac{\partial \alpha}{\partial s}(s, t)=$ $-\operatorname{sgn}(\kappa) g(s, t) J \frac{\partial \alpha}{\partial t}(s, t)$ with a function $g$ satisfying

$$
\frac{\partial^{2} g}{\partial t^{2}}(s, t)+g(s, t) \operatorname{HR}\left(\frac{\partial \alpha}{\partial t}(s, t)\right) \equiv 0, \quad g(s, 0)=1, \quad \frac{\partial g}{\partial t}(s, 0)=-|\kappa|,
$$

where HR(v) denotes the holomorphic sectional curvature of the line spanned by a unit vector $v$. Thus we have

$$
\begin{aligned}
& \nabla_{\frac{\partial \alpha}{\partial t}} \frac{\partial \alpha}{\partial t}=0, \\
& \nabla_{\frac{\partial \alpha}{\partial s} \frac{\partial \alpha}{\partial t}}=\nabla_{\frac{\partial \alpha}{\partial t}} \frac{\partial \alpha}{\partial s}=-\operatorname{sgn}(\kappa) \nabla_{\frac{\partial \alpha}{\partial t}}\left(g J \frac{\partial \alpha}{\partial t}\right)=-\operatorname{sgn}(\kappa) \frac{\partial g}{\partial t} J \frac{\partial \alpha}{\partial t}, \\
& \nabla_{\frac{\partial \alpha}{\partial s}} \frac{\partial \alpha}{\partial s}=-\operatorname{sgn}(\kappa) \nabla_{\frac{\partial \alpha}{\partial s}}\left(g J \frac{\partial \alpha}{\partial t}\right)=-\operatorname{sgn}(\kappa)\left(\frac{\partial g}{\partial s} J \frac{\partial \alpha}{\partial t}+g J \nabla_{\frac{\partial \alpha}{\partial s}} \frac{\partial \alpha}{\partial t}\right) .
\end{aligned}
$$

Hence these vectors $\nabla_{\frac{\partial \alpha}{\partial t}} \frac{\partial \alpha}{\partial t}, \nabla_{\frac{\partial \alpha}{\partial s}} \frac{\partial \alpha}{\partial t}, \nabla_{\frac{\partial \alpha}{\partial s}} \frac{\partial \alpha}{\partial s}$ are contained in the tangent space of a surface formed by $\alpha$, and the surface is totally geodesic.

We call a point $\sigma\left(t_{0}\right)$ a $\mathbf{B}_{\kappa}$-trajectory focal point of $\sigma(0)$ if there is a Jacobi field along $\sigma$ associated with a $\mathbf{B}_{\kappa}$-trajectory which vanishes at $t_{0}$, and call the value $t_{0} /\left\|\sigma^{\prime}\right\|$ a $\mathbf{B}_{\kappa}$-trajectory focal value of $\sigma(0)$. The minimum positive $\mathbf{B}_{\kappa}$-trajectory focal value is said to be the first $\mathbf{B}_{\kappa^{-}}$ trajectory focal value of $\sigma(0)$, and is denoted by $t_{f}(\sigma(0) ; \sigma, \kappa)$ or $t_{f}(\sigma(0) ; \kappa)$. In case every point $\sigma(t), t>0$ is not a $\mathbf{B}_{\kappa}$-trajectory focal point of $\sigma(0)$ we put $t_{f}(\sigma(0) ; \sigma, \kappa)=\infty$. We denote the maximum negative $\mathbf{B}_{\kappa}$-trajectory focal value by $-t_{n}(\sigma(0) ; \sigma, \kappa)$. We also put $t_{n}(\sigma(0) ; \sigma, \kappa)=\infty$ if there are no negative $\mathbf{B}_{\kappa}$-trajectory focal values.

We here study $\mathbf{B}_{\kappa}$-trajectory focal values for a complex space form $\mathbf{C} M^{n}(c)$, which is a complex projective space $\mathbf{C} P^{n}(c)$ of constant holomorphic sectional curvature $c$, a complex Euclidean space $\mathbf{C}^{n}$ or a complex hyperbolic space $\mathbf{C} H^{n}(c)$ of constant holomorphic sectional curvature $c$ according $c$ is positive, null or negative. On a complex Euclidean space $\mathbf{C}^{n}$, a trajectory for $\mathbf{B}_{\kappa}$ is a circle of radius $1 /|\kappa|$ in the sense of Euclidean geometry. Thus geodesics associated with a trajectory meet at its center, hence the first $\mathbf{B}_{\kappa}$-trajectory focal value is $1 /|\kappa|$ and has no negative $\mathbf{B}_{\kappa}$-trajectory focal values. 
On a complex projective space $\mathbf{C} P^{n}(c)$, every trajectory lies on some totally geodesic sphere $\mathbf{C} P^{1}(c)$ and is a small circle. If we regard this as a latitude line, geodesics associated with this trajectory meet at poles of $\mathbf{C} P^{1}(c)$ which are the center of this small circle and its anti-podal point. Hence the first $\mathbf{B}_{\kappa}$-trajectory focal value is $(1 / \sqrt{c}) \tan ^{-1}(\sqrt{c} /|\kappa|)$ and the maximum negative $\mathbf{B}_{\kappa}$-trajectory focal value is $-(1 / \sqrt{c})\left\{\pi-\tan ^{-1}(\sqrt{c} /|\kappa|)\right\}$. A Jacobi field along a normal geodesic $\sigma$ associated with a trajectory for $\mathbf{B}_{\kappa}$ on $\mathbf{C} P^{n}(c)$ is of the form

$$
Y_{c}(t)=a t \dot{\sigma}(t)+\left\{-\operatorname{sgn}(\kappa) \cos \sqrt{c} t+\frac{\kappa}{\sqrt{c}} \sin \sqrt{c} t\right\} J \dot{\sigma}(t)
$$

with a constant $a$.

On a complex hyperbolic space $\mathbf{C} H^{n}(c)$, every trajectory lies on some totally geodesic real hyperbolic plane $\mathbf{C} H^{1}(c)$. A Jacobi field along a normal geodesic $\sigma$ associated with a trajectory for $\mathbf{B}_{\kappa}$ on $\mathbf{C} H^{n}(c)$ is of the form

$$
Y_{c}(t)=\operatorname{at} \dot{\sigma}(t)+\left\{-\operatorname{sgn}(\kappa) \cosh \sqrt{|c|} t+\frac{\kappa}{\sqrt{|c|}} \sinh \sqrt{|c|} t J \dot{\sigma}(t)\right.
$$

with a constant $a$. Therefore, if $|\kappa| \leq \sqrt{|c|}$, there are no $\mathbf{B}_{\kappa}$-trajectory focal points, and if $|\kappa|>\sqrt{|c|}$, the first $\mathbf{B}_{\kappa}$-trajectory focal value is $(1 / 2 \sqrt{|c|}) \log (\sqrt{|c|}+|\kappa|) /(|\kappa|-\sqrt{|c|})$ and there is no negative $\mathbf{B}_{\kappa}$-trajectory focal values.

We denote by $t_{f}(c ; \kappa)$ and $-t_{n}(c ; \kappa)$ the first $\mathbf{B}_{\kappa}$-trajectory focal value and the maximum negative $\mathbf{B}_{\kappa}$-trajectory focal value on a complex space form of constant holomorphic sectional curvature $c$. Then we see

$$
\begin{aligned}
& t_{f}(c ; \kappa)= \begin{cases}\frac{1}{\sqrt{c}} \tan ^{-1} \frac{\sqrt{c}}{|\kappa|}, & \text { if } c>0, \\
\frac{1}{|\kappa|}, & \text { if } c=0, \\
\frac{1}{2 \sqrt{|c|}} \log \frac{\sqrt{|c|}+|\kappa|}{|\kappa|-\sqrt{|c|},} & \text { if } c<0 \text { and }|\kappa|>\sqrt{|c|}, \\
\infty, & \text { if } c<0 \text { and }|\kappa| \leq \sqrt{|c|},\end{cases} \\
& t_{n}(c ; \kappa)= \begin{cases}\frac{1}{\sqrt{c}}\left(\pi-\tan ^{-1} \frac{\sqrt{c}}{|\kappa|}\right), & \text { if } c>0, \\
\infty, & \text { if } c \leq 0 .\end{cases}
\end{aligned}
$$

We here give comparison results on first $\mathbf{B}_{\kappa}$-focal values.

Proposition 1. Let $M$ be a Kähler manifold and $\sigma$ be a normal geodesic on $M$. If the sectional curvatures of 2-planes spanned by $\dot{\sigma}(t)$ and a vector orthogonal to $\dot{\sigma}(t)$ are not greater than $c$ for $0 \leq t \leq t_{f}(\sigma(0) ; \sigma, \kappa)$, then we have the following:

(1) $t_{f}(\sigma(0) ; \sigma, \kappa) \geq t_{f}(c ; \kappa)$.

(2) Let $Y$ be a Jacobi field along a geodesic $\sigma$ which is associated with a trajectory for $\mathbf{B}_{\kappa}$, and $\hat{Y}$ be a Jacobi field along a normal geodesic $\hat{\sigma}$ which is associated with a trajectory for $\mathbf{B}_{\kappa}$ on a simply connected surface $\hat{M}=\mathbf{C} M^{1}$ (c) of constant sectional curvature c. 
If $Y$ is orthogonal to $\dot{\sigma}$ and $\hat{Y}$ is orthogonal to $\dot{\hat{\sigma}}$, then $\|Y(t)\| \geq\|\hat{Y}(t)\|$ for every $t$ with $0 \leq t \leq t_{f}(c ; \kappa)$. The equality $\left\|Y\left(t_{0}\right)\right\|=\left\|\hat{Y}\left(t_{0}\right)\right\|\left(0<t_{0} \leq t_{f}(c ; \kappa)\right)$ holds if and only if $Y(t)$ is parallel to $J \dot{\sigma}(t)$ and the holomorphic sectional curvature of the line spanned by $\dot{\sigma}(t)$ is equal to $c$ for $0 \leq t \leq t_{0}$.

Proof. For the sake of simplicity, we suppose $\kappa>0$. We denote $Y$ and $\hat{Y}$ by $Y=h E$ and $\hat{Y}=-g J \dot{\hat{\sigma}}$ with functions $g, h$ and a vector field $E$ along $\sigma$ satisfying $h(0)=g(0)=$ $1,\|E\|=1$ and $\langle E, \dot{\sigma}\rangle=0$. As $\left\langle\nabla_{\dot{\sigma}} E, E\right\rangle=0$ and $\left\langle\nabla_{\dot{\sigma}} \nabla_{\dot{\sigma}} E, E\right\rangle=-\left\|\nabla_{\dot{\sigma}} E\right\|^{2}$, we see

$$
h^{\prime \prime}+h\left(\langle R(E, \dot{\sigma}) \dot{\sigma}, E\rangle-\left\|\nabla_{\dot{\sigma}} E\right\|^{2}\right)=0 .
$$

Therefore, for $0 \leq t<\min \left\{t_{f}(\sigma(0) ; \sigma, \kappa), t_{f}(c ; \kappa)\right\}$ we have

$$
\begin{aligned}
\left(h^{\prime} g-h g^{\prime}\right)^{\prime} & =h g\left(\left\|\nabla_{\dot{\sigma}} E\right\|^{2}-\langle R(E, \dot{\sigma}) \dot{\sigma}, E\rangle+c\right) \\
& \geq h g(c-\langle R(E, \dot{\sigma}) \dot{\sigma}, E\rangle) \geq 0 .
\end{aligned}
$$

By the definition of Jacobi fields associated with trajectories for $\mathbf{B}_{\kappa}$, we see $h^{\prime}(0)=g^{\prime}(0)=$ $-\kappa$ and $\left(h^{\prime} g-h g^{\prime}\right)(0)=0$. Therefore we find $(h / g)^{\prime} \geq 0$, hence $h \geq g$.

The equality $h\left(t_{0}\right)=g\left(t_{0}\right)$ at some point $0<t_{0}<t_{f}(c ; \kappa)$ holds if and only if $\nabla_{\dot{\sigma}} E \equiv 0$ and $\langle R(E, \dot{\sigma}) \dot{\sigma}, E\rangle \equiv c$ for $0 \leq t \leq t_{0}$. This is the case that $E=-J \dot{\sigma}$ and the holomorphic sectional curvature of the complex line spanned by $\dot{\sigma}(t)$ is $c$ for $0 \leq t \leq t_{0}$.

The proof of Proposition 1 also guarantees the following.

PROPOSITION 2. Let $M$ be a Kähler manifold and $\sigma$ be a normal geodesic on $M$. If the sectional curvatures of 2-planes spanned by $\dot{\sigma}(t)$ and a vector orthogonal to $\dot{\sigma}(t)$ are not greater than $c$ for $t<0$, then $t_{n}(\sigma(0) ; \sigma, \kappa) \geq t_{n}(c ; \kappa)$.

For two unit tangent vectors $v, w \in U M(v \neq \pm w)$, we denote by $\operatorname{Riem}(v, w)$ the sectional curvature of the plane spanned by $v, w$. In view of the values $t_{f}(c ; \kappa)$ of the first $\mathbf{B}_{\kappa^{-}}$ focal value on a complex space form and $t_{n}(c ; \kappa)$, these propositions guarantee the following.

COROLLARY 1. On a Kähler manifold $M$ whose sectional curvature satisfies Riem $\leq$ $c$ with some nonpositive constant $c$, every variation of geodesics associated with a trajectory for $\mathbf{B}_{\kappa}$ forms an immersed surface without singularities if $|\kappa| \leq \sqrt{|c|}$.

\section{Crescents and Bow-shapes}

In Riemannian geometry, a comparison theorem for triangles plays quite an important role. This Toponogov theorem says that triangles on a manifold of large sectional curvature are fatter than triangles on a manifold of small sectional curvature. We here prepare a corresponding result for trajectories. As a substitute for a triangle we consider a bow-shape, which is consisted of a trajectory segment and a kind of geodesic segment.

A crescent for $\mathbf{B}_{\kappa}$ on a Kähler manifold $M$ is a pair $\mathcal{C}=(\gamma, \tau)$ of a trajectory segment $\gamma:[0, L] \rightarrow M$ for $\mathbf{B}_{\kappa}$ and a nonnegative function $\tau:[0, L] \rightarrow[0, \infty)$ satisfying $\tau(0)=$ 
$\tau(L)=0$ and $0 \leq \tau(s)<t_{f}(\gamma(s) ; \kappa)$ for every $s$. For a crescent $\mathcal{C}=(\gamma, \tau)$ we call $\gamma$ the arc of $\mathcal{C}$. If $\alpha:[0, L] \times \mathbf{R} \rightarrow M$ is the variation of normal geodesics associated with $\gamma$, we call the set $\operatorname{Rep}(\mathcal{C})=\{\alpha(s, t) \mid 0 \leq s \leq L, 0 \leq t \leq \tau(s)\}$ the represented shape of $\mathcal{C}$. We denote by $\rho_{\mathcal{C}}$ the curve $[0, L] \ni s \mapsto \alpha(s, \tau(s)) \in M$. If a crescent $\mathcal{B}$ with $\operatorname{arc} \gamma$ has the minimum length of periphery among crescents $\mathcal{C}$ with $\operatorname{arc} \gamma$, that is, length $\left(\rho_{\mathcal{C}}\right) \geq$ length $\left(\rho_{\mathcal{B}}\right)$, we shall call it a bow-shape with arc $\gamma$, and call the curve $\rho_{\mathcal{B}}$ the bow-string of $\mathcal{B}$. As a matter of course, a bow-shape does not necessarily exists for every trajectory segment. Roughly speaking, if a bow-string $\rho_{\mathcal{B}}$ exists for a trajectory segment $\gamma$ its image is an image of minimal geodesic joining the origin and terminus of $\gamma$ on a part of a surface formed by a variation of geodesics associated with $\gamma$. We here make mention of bow-shapes on a complex space form $\mathbf{C} M^{n}(c)$ of constant holomorphic sectional curvature $c$. For bow-strings we sometimes call their images also bow-strings.

Example 1. On a complex Euclidean space $\mathbf{C}^{n}$, for a trajectory segment $\gamma$ for $\mathbf{B}_{\kappa}$ with length $(\gamma)<\pi /|\kappa|$, we have a unique bow-shape $\mathcal{B}$ whose bow-string $\rho_{\mathcal{B}}$ is an image of a geodesic segment and satisfies

$$
\text { length }\left(\rho_{\mathcal{B}}\right)=\frac{2}{|\kappa|} \sin \left(\frac{1}{2}|\kappa| \text { length }(\gamma)\right) .
$$

The image of this bow-shape lies on a totally geodesic $\mathbf{C}^{1}$. But if length $(\gamma) \geq \pi /|\kappa|$, there does not exist bow-shapes with arc $\gamma$.

EXAMPLE 2. On a complex projective space $\mathbf{C} P^{n}(c)$, for a trajectory segment $\gamma$ for $\mathbf{B}_{\kappa}$ with length $(\gamma)<\pi / \sqrt{\kappa^{2}+c}$, we have a unique bow-shape $\mathcal{B}$ whose bow-string $\rho_{\mathcal{B}}$ is an image of a geodesic segment and satisfies

$$
\sqrt{c} \sin \left(\frac{1}{2} \sqrt{\kappa^{2}+c} \text { length }(\gamma)\right)=\sqrt{\kappa^{2}+c} \sin \left(\frac{1}{2} \sqrt{c} \text { length }\left(\rho_{\mathcal{B}}\right)\right) .
$$

In particular, we see length $\left(\rho_{\mathcal{B}}\right) \leq(2 / \sqrt{c}) \sin ^{-1} \sqrt{c /\left(\kappa^{2}+c\right)}$. The image of this bow-shape lies on some totally geodesic standard sphere $\mathbf{C} P^{1}(c)$, and its periphery consists of a part of a small circle and a part of a great circle.

For a trajectory segment $\gamma$ with length $(\gamma) \geq \pi / \sqrt{\kappa^{2}+c}$, there does not exist bowshapes with arc $\gamma$.

EXAMPLE 3. Let $\gamma$ be a trajectory segment for $\mathbf{B}_{\kappa}$ on a complex hyperbolic space $\mathbf{C} H^{n}(c)$

(1) When $|\kappa| \leq \sqrt{|c|}$, we have a unique bow-shape $\mathcal{B}$ whose bow-string $\rho_{\mathcal{B}}$ is an image of a geodesic segment and satisfies

$$
\left\{\begin{array}{cc}
\sqrt{|c|} \sinh \left(\sqrt{|c|-\kappa^{2}} \text { length }(\gamma) / 2\right) & \text { if }|\kappa|<\sqrt{|c|}, \\
=\sqrt{|c|-\kappa^{2}} \sinh \left(\sqrt{|c|} \text { length }\left(\rho_{\mathcal{B}}\right) / 2\right), & \\
\sqrt{|c|} \text { length }(\gamma)=2 \sinh \left(\sqrt{|c|} \text { length }\left(\rho_{\mathcal{B}}\right) / 2\right), & \text { if } \kappa= \pm \sqrt{|c|} .
\end{array}\right.
$$


(2) When $|\kappa|>\sqrt{|c|}$, if length $(\gamma)<\pi / \sqrt{\kappa^{2}+c}$, we have a unique bow-shape $\mathcal{B}$ whose bow-string $\rho_{\mathcal{B}}$ is an image of a geodesic segment and satisfies

$$
\sqrt{|c|} \sin \left(\frac{1}{2} \sqrt{\kappa^{2}+c} \text { length }(\gamma)\right)=\sqrt{\kappa^{2}+c} \sinh \left(\frac{1}{2} \sqrt{|c|} \operatorname{length}\left(\rho_{\mathcal{B}}\right)\right) .
$$

In particular, we see length $\left.\left(\rho_{\mathcal{B}}\right) \leq(2 / \sqrt{|c|}) \log (|\kappa|+\sqrt{|c|}) / \sqrt{\kappa^{2}+c}\right)$. But if length $(\gamma) \geq$ $\pi / \sqrt{\kappa^{2}+c}$, there does not exist bow-shapes with arc $\gamma$.

(3) Every image of above bow-shapes is contained in some totally geodesic real hyperbolic plane $\mathrm{CH}^{1}(c)$.

Needless to say that on $\mathbf{C} \mathbf{M}^{n}(c)$ the represented shape of each bow-shape is simply connected. As a matter of fact, it is an image of a simply connected subset of the tangent space through the exponential map. For example, when $\mathcal{B}$ is a bow-shape on $\mathbf{C}^{n}$ whose $\operatorname{arc}$ $\gamma:[0, \ell] \rightarrow \mathbf{C}^{n}$ is a trajectory for $\mathbf{B}_{\kappa}$, then we see

$$
\left.\operatorname{Rep}(\mathcal{B})=\exp _{\gamma(0)}\left(\begin{array}{l|l}
v(u, \theta) \in T_{\gamma(0)} \mathbf{C}^{n} & \begin{array}{l}
0 \leqq \theta \leqq|\kappa| \ell / 2 \\
0 \leqq u \leqq(2 /|\kappa|) \sin \theta
\end{array}
\end{array}\right\}\right)
$$

where $v(u, \theta)=u \cos \theta \dot{\gamma}(0)+\operatorname{sgn}(\kappa) u \sin \theta J \dot{\gamma}(0)$.

We now give a comparison theorem on bow-shapes.

THEOREM 1. Let $M$ be a Kähler manifold satisfying Riem $\leq c$ with a constant $c$. If $a$ $\mathbf{B}_{\kappa}$-crescent $\mathcal{C}=(\gamma, \tau)$ satisfies

i) length $(\gamma)<\pi / \sqrt{\kappa^{2}+c}$ when $\kappa^{2}+c>0$,

ii) $\tau(s)<t_{f}(c ; \kappa)$ for every $s$,

then length $\left(\rho_{\mathcal{C}}\right)$ is not smaller than the length length $\left(\rho_{\mathcal{B}}\right)$ of a bow-string of a $\mathbf{B}_{\kappa}$-bow-shape $\mathcal{B}$ on a complex space form $\mathbf{C} M^{n}(c)$ whose length of arc is length $(\gamma)$.

The equality length $\left(\rho_{\mathcal{C}}\right)=$ length $\left(\rho_{\mathcal{B}}\right)$ holds if and only if the represented shape of $\mathcal{C}$ is complex analytically isometrically immersed image of the represented shape of $\mathcal{B}$ and is totally geodesic. In this case, it is a bow-shape with arc $\gamma$.

Proof. Put $L=$ length $(\gamma)$. We take a trajectory segment $\hat{\gamma}$ on $\hat{M}=\mathbf{C} M^{n}(c)$ satisfying length $(\hat{\gamma})=L$, and consider a crescent $\hat{\mathcal{C}}=(\hat{\gamma}, \tau)$. Let $\alpha:[0, L] \times \mathbf{R} \rightarrow M$ and $\hat{\alpha}:[0, L] \times \mathbf{R} \rightarrow \hat{M}$ be variations of normal geodesics associated with $\gamma$ and $\hat{\gamma}$ respectively. As we have $\left\langle\frac{\partial \alpha}{\partial s}, \frac{\partial \alpha}{\partial t}\right\rangle=\left\langle\frac{\partial \hat{\alpha}}{\partial s}, \frac{\partial \hat{\alpha}}{\partial t}\right\rangle=0$, we find by Proposition 1 that

$$
\begin{aligned}
\operatorname{length}\left(\rho_{\mathcal{C}}\right) & =\int_{0}^{L}\left\|\rho_{\mathcal{C}}^{\prime}(s)\right\| d s=\int_{0}^{L} \sqrt{\left\|\frac{\partial \alpha}{\partial s}(s, \tau(s))\right\|^{2}+\tau^{\prime}(s)^{2}} d s \\
& \geq \int_{0}^{L} \sqrt{\left\|\frac{\partial \hat{\alpha}}{\partial s}(s, \tau(s))\right\|^{2}+\tau^{\prime}(s)^{2}} d s=\operatorname{length}\left(\rho_{\hat{\mathcal{C}}}\right) \\
& \geq \operatorname{length}\left(\rho_{\mathcal{B}}\right) .
\end{aligned}
$$


The equality holds if and only if $\left\|\frac{\partial \alpha}{\partial s}(s, \tau(s))\right\|=\left\|\frac{\partial \hat{\alpha}}{\partial s}(s, \tau(s))\right\|$ holds for every $s$ and $\hat{\mathcal{C}}=\mathcal{B}$. Again by Proposition 1, we see it is the case that the holomorphic sectional curvature of the line spanned by $\frac{\partial \alpha}{\partial s}(s, t)$ is $c$ for every $(s, t)$ with $0 \leq t \leq \tau(s)$.

REMARK. The above proof also shows that the length of smooth curve on a surface $A=\left\{\alpha(s, t) \mid 0 \leq s \leq L, 0 \leq t \leq t_{f}(c ; \kappa)\right\}$ joining $\gamma(0)$ and $\gamma(L)$ is not smaller than the length of bow-string length $\left(\rho_{\mathcal{B}}\right)$ of a bow-shape $\mathcal{B}$ on $\mathbf{C} M^{n}(c)$.

If a crescent $\widetilde{\mathcal{C}}=(\gamma, \tilde{\tau})$ on $M$ does not lie on $A$, as we have $s_{0}$ with $\tau\left(s_{0}\right)>t_{f}(c ; \kappa)$, we find length $\left(\rho_{\mathcal{C}}\right) \geq 2 t_{f}(c ; \kappa)>$ length $\left(\rho_{\mathcal{B}}\right)$. In particular, if there is a crescent $\widetilde{\mathcal{C}}$ on $M$ with length $\left(\rho_{\widetilde{\mathcal{C}}}\right)=$ length $\left(\rho_{\mathcal{B}}\right)$, then it should lie on $A$.

When $\kappa^{2}+c \leq 0$, or when $\kappa^{2}+c>0$ and $L<\pi / \sqrt{\kappa^{2}+c}$, we denote by $\ell(\kappa, L ; c)$ the length of a bow-string of a bow-shape for $\mathbf{B}_{\kappa}$ on a complex space form $\mathbf{C} M^{n}(c)$ whose length of arc is $L$. We set $\delta(\kappa, c)=\pi / \sqrt{\kappa^{2}+c}$ when $\kappa^{2}+c>0$ and $\delta(\kappa, c)=\infty$ when $\kappa^{2}+c \leq 0$. By standing another point of view, we can conclude the following.

PROPOSITION 3. Let $M$ be a Kähler manifold satisfying Riem $\leq c$ with a constant $c$. If a bow-shape $\mathcal{C}$ for $\mathbf{B}_{\kappa}$ on $M$ satisfies length $\left(\rho_{\mathcal{C}}\right)=\ell(\kappa, L ; c)$ for some positive $L$ satisfying $L<\delta(\kappa, c)$, then the length of the arc of $\mathcal{C}$ is not longer than $L$.

If a trajectory segment $\gamma$ for $\mathbf{B}_{\kappa}$ on a Kähler manifold satisfying Riem $\leq c$ has a bowshape $\mathcal{B}$ with arc $\gamma$ and length $\left(\rho_{\mathcal{B}}\right)=\ell(\kappa$, length $(\gamma) ; c)$, then the holomorphic sectional curvature of the complex line spaned by $\dot{\gamma}$ is $c$. Taking account of this we shall say that a trajectory $\gamma$ for $\mathbf{B}_{\kappa}(\kappa \neq 0)$ on a Kähler manifold is of $c$-space type if there exists a sequence $\left\{s_{j}\right\}_{j=-\infty}^{\infty}$ satisfying the following conditions:

i) $\lim _{j \rightarrow \infty} s_{j}=\delta(\kappa, c)$ and $\lim _{j \rightarrow-\infty} s_{j}=-\delta(\kappa, c)$,

ii) for each $s_{j}\left(s_{j} \neq 0\right)$, the trajectory segment $\left.\gamma\right|_{I_{j}}$, which is a restriction of $\gamma$ on the interval $I_{j}$, has a bow-shape $\mathcal{B}_{j}=\left(\left.\gamma\right|_{I_{j}}, \tau_{j}\right)$ with length $\left(\rho_{\mathcal{B}_{j}}\right)=\ell\left(\kappa,\left|s_{j}\right| ; c\right)$, where $I_{j}=\left[0, s_{j}\right]$ for $s_{j}>0$ and $I_{j}=\left[s_{j}, 0\right]$ for $s_{j}<0$.

It is needless to say that every trajectory on a complex space form $\mathbf{C} M^{n}(c)$ is of $c$-space type. A trajectory $\gamma$ for $\mathbf{B}_{\kappa}$ on $\mathbf{C} M^{n}(c)$ is closed if and only if $\kappa^{2}+c>0$. In this case its minimal period length $(\gamma)$ is $2 \pi / \sqrt{\kappa^{2}+c}=2 \delta(\kappa, c)$ and the geodesic with initial vector $\operatorname{sgn}(\kappa) J \dot{\gamma}(0)$ goes through the point $\gamma(\delta(\kappa, c))$ (see [1]).

As a direct consequence of Theorem 1 we have

COROLlary 2. Let $M$ be a Kähler manifold satisfying Riem $\leq c$.

(1) If $b>c$, there does not exist a trajectory of $b$-space type.

(2) If $\kappa^{2}+c>0$, every trajectory $\gamma$ of c-space type for $\mathbf{B}_{\kappa}$ is closed and length $(\gamma)=$ $2 \pi / \sqrt{\kappa^{2}+c}$. For a variation $\alpha$ of normal geodesics associated with $\gamma$, the interior

$$
\mathcal{F}_{\alpha}=\left\{\alpha(s, t)|| s \mid \leq \delta(\kappa ; c), 0 \leq t \leq t_{f}(c ; \kappa)\right\}
$$

is totally geodesic, complex and of constant curvature $c$. 
Proof. (1) Since $\ell(\kappa, L ; b)<\ell(\kappa, L ; c)$ when $b>c$, the first assertion is trivial by Theorem 1.

(2) If there is a crescent $\mathcal{C}=(\beta, \tau)$ on $M$ with length $(\beta)<\delta(\kappa, c)$ and length $\left(\rho_{\mathcal{C}}\right)$ $=\ell(\kappa$, length $(\beta) ; c)$, then by Theorem 1 and Remark we see the represented shape of $\mathcal{C}$ is totally geodesic and of holomorphic sectional curvature $c$. We put $v_{j}=\dot{\rho}_{\mathcal{C}_{j}}(0) /\left\|\dot{\rho}_{\mathcal{C}_{j}}(0)\right\| \in$ $U_{\gamma\left(s_{j}\right)} M$. Since the represented shape of $\mathcal{B}_{j}$ is complex analytically isometrically immersed image of the represented shape of a bow-shape on $\mathbf{C} M^{n}(c)$ whose length of $\operatorname{arc}$ is $\left|s_{j}\right|$, we find that $\lim _{j \rightarrow \infty} v_{j}=\lim _{j \rightarrow-\infty} v_{j}=\operatorname{sgn}(\kappa) J \dot{\gamma}(0)$. This shows $\gamma(\delta(\kappa, c))=\gamma(-\delta(\kappa, c))$, hence $\gamma$ is closed and length $(\gamma)=2 \delta(\kappa, c)$.

In the last stage we make mention of bow-shapes on a product of complex space forms. On a product $M=M_{1} \times M_{2}$ of Kähler manifolds $M_{i}$, every trajectory $\gamma$ is of the form $\gamma(t)=\left(\gamma_{1}\left(\lambda_{1} t\right), \gamma_{2}\left(\lambda_{2} t\right)\right)$. Here, $\lambda_{1}, \lambda_{2}$ are nonnegative constants with $\lambda_{1}^{2}+\lambda_{2}^{2}=1$, and $\gamma_{i}$ is a trajectory for $\mathbf{B}_{\kappa} / \lambda_{i}$ on $M_{i}$ when $\lambda_{i}>0$ and is a point curve on $M_{i}$ when $\lambda_{i}=0$ (see $[3,4])$. One can easily compute the length of bow-string on a product of complex space forms. For example, on a product $\mathbf{C} P^{n_{1}}\left(c_{1}\right) \times \cdots \times \mathbf{C} P^{n_{p}}\left(c_{p}\right)$ of complex projective spaces, a trajectory segment $\gamma$ of the form $\gamma(t)=\left(\gamma_{1}\left(\lambda_{1} t\right), \cdots, \gamma_{p}\left(\lambda_{p} t\right)\right)$ for $\mathbf{B}_{\kappa}$ with nonnegative constants $\lambda_{1}, \cdots, \lambda_{p}$ satisfying $\sum_{i=1}^{p} \lambda_{i}^{2}=1$ has a bow-shape if

$$
\text { length }(\gamma)<\min \left\{\pi / \sqrt{\kappa^{2}+c_{i} \lambda_{i}^{2}} \mid \lambda_{i} \neq 0,1 \leq i \leq p\right\} .
$$

The length of its bow-string is given by $\sqrt{\sum_{i=1}^{p} d_{i}^{2}}$ with $d_{i}$ satisfying

$$
\left.\lambda_{i} \sqrt{c_{i}} \sin \left(\sqrt{\kappa^{2}+c_{i} \lambda_{i}^{2}} \text { length }(\gamma) / 2\right)=\sqrt{\kappa^{2}+c_{i} \lambda_{i}^{2}} \sin \left(\sqrt{c_{i}} d_{i} / 2\right)\right) .
$$

We here consider a subset $\mathcal{S}_{x}(c)$ of the unit tangent space $U_{x} M$ given by

$$
\mathcal{S}_{x}(c)=\left\{\begin{array}{l|l}
v \in U_{x} M & \begin{array}{c}
\text { there is a positive } \varepsilon \text { such that for every } \\
\kappa \text { with } 0<|\kappa| \leq \varepsilon \text { the trajectory for } \mathbf{B}_{\kappa} \\
\text { with initial vector } v \text { is of } c \text {-space type }
\end{array}
\end{array}\right\} .
$$

For a complex space form $\mathbf{C} M^{n}(c)$ we see $U_{x} \mathbf{C} M^{n}(c)=\mathcal{S}_{x}(c)$ at each point, and for a product of complex space forms $M=\mathbf{C} M^{n_{1}}\left(c_{1}\right) \times \cdots \times \mathbf{C} M^{n_{p}}\left(c_{p}\right)$, we see that $\mathcal{S}_{x}(c)$ is either an empty set or a disjoint sum of spheres; $\mathcal{S}_{x}(c)=S^{2 n_{i_{1}}-1}+\cdots+S^{2 n_{i_{q}}-1}$, where $c=c_{i_{j}}$ for $1 \leq j \leq q, c_{i} \neq c$ for $i \neq i_{j}$. Here, if we denote $x \in M$ by $\left(x_{1}, \cdots, x_{p}\right)$, the set $S^{2 n_{i_{j}}}$ corresponds to $U_{x_{i j}} \mathbf{C} M^{n_{i j}}(c)$. For a Hermitian symmetric space $M$ of rank $r$, it was pointed out by Ikawa[10] that every trajectory lies on a totally geodesic $r$-product $\mathbf{C} M^{1}(c) \times \cdots \times \mathbf{C} M^{1}(c)$, where $c$ is the maximum sectional curvature when $M$ is of compact type and is the minimum sectional curvature when $M$ is of noncompact type. We hence see that for this $c$ the set $S_{x}(c)$ contains a $r$-sum $S^{1}+\cdots+S^{1}$ of circles $S^{1}$. 
COROLlaRY 3. Let $M$ be a simply connected Kähler manifold of Riem $\leq c$ for some nonnegative $c$. If $\mathcal{S}_{x}(c) \neq \emptyset$ at some point $x$, then $M$ contains a totally geodesic $\mathbf{C} M^{1}(c)$.

PROOF. For $v \in \mathcal{S}_{x}(c)$ we denote by $\gamma_{\kappa}(0<|\kappa|<\varepsilon)$ a trajectory of $c$-space type for $\mathbf{B}_{\kappa}$ with $\dot{\gamma}_{\kappa}(0)=v$, and by $\alpha_{\kappa}$ the variation of normal geodesics associated with $\gamma_{\kappa}$. Since $M$ satisfies Riem $\leq c$, we see $\mathcal{F}_{\alpha_{\kappa}}$ is totally geodesic and of constant curvature $c$. Moreover, as $M$ is simply connected, the condition that $\gamma_{\kappa}$ is of $c$-space type guarantees that $\mathcal{F}_{\alpha_{\kappa}}$ is contained in the inside of the geodesic ball centered at $\gamma(0)$ whose radius is the injectivity radius at $\gamma(0)$. Thus $\mathcal{F}_{\alpha_{\kappa}}$ is an image of a simply connected subset of $\{a \dot{\gamma}(0)+b J \dot{\gamma}(0) \mid a \in \mathbf{R}, b>0\}$ through the exponential map $\exp _{\gamma(0)}$ when $\kappa>0$ and is an image of a simply connected subset of $\{a \dot{\gamma}(0)+b J \dot{\gamma}(0) \mid a \in \mathbf{R}, b<0\}$ through this exponential map when $\kappa<0$. We hence find that $\mathcal{F}_{\alpha_{\kappa}}$ is simply connected and that $\mathcal{F}_{\alpha_{\kappa_{1}}} \supset \mathcal{F}_{\alpha_{\kappa_{2}}}$ if $0<\kappa_{1}<\kappa_{2}<\varepsilon$ or $0>\kappa_{1}>\kappa_{2}>-\varepsilon$. Therefore we see $\mathcal{F}=\bigcup_{0<|\kappa|<\varepsilon} \mathcal{F}_{\alpha_{\kappa}}$ is totally geodesic and is complex analytically isometric to $\mathbf{C} M^{1}(c) \backslash\left\{\right.$ image of a geodesic on $\left.\mathbf{C} M^{1}(c)\right\}$. Since the topological closure

$$
\overline{\mathcal{F}}=\mathcal{F} \cup\{\text { the image of the geodesic with initial vector } v\}
$$

of $\mathcal{F}$ is of constant curvature $c$, we see it is complex analytically isometric to $\mathbf{C} M^{1}(c)$.

If we restrict ourselves on Hermitian symmetric spaces, as every trajectory lies on a totally geodesic $r$-product of $\mathbf{C} M^{1}$, s, the following is trivial.

COROLlaRY 4. If a Hermitian symmetric space $M$ satisfies $\mathcal{S}_{x}(c)=U_{x} M$ for some $c$, then $M$ is $\mathbf{C} M^{n}(c)$.

\section{References}

[ 1 ] T. ADACHI, Kähler magnetic flows on a manifold of constant holomorphic sectional curvature, Tokyo J. Math. 18 (1995), 473-483.

[2] T. ADACHI, A comparison theorem on magnetic Jacobi fields, Proc. Edinburgh Math. Soc. 40 (1997), $293-308$.

[3] T. ADACHI, Kähler magnetic flows for a product of complex space forms, Topology and its application 146147 (2005), 329-338.

[ 4 ] T. ADACHI, Rank of a Hermitian symmetric space of noncompact type and Kähler magnetic fields, preprint.

[ 5 ] T. Adachi, S. Maeda and S. UdagaWA, Simpleness and closedness of circles in compact Hermitian symmetric spaces, Tsukuba J. Math. 24 (2000), 1-13.

[6] M. L. Bialy, Rigidity for periodic magnetic fields, Ergod. Th. Dynam. Sys. 20 (2000), 1619-1626.

[ 7 ] J. CheEgER and D. EBIN, Comparison theorems in Riemannian geometry, North-Holland, 1975.

[ 8 ] N. GoudA, Magnetic flows of Anosov type, Tôhoku Math. J. 49 (1997), 165-183.

[9] N. GoudA, The theorem of E. Hopf under uniform magnetic fields, J. Math. Soc. Japan 50 (1998), 767-779.

[10] O. IKAWA, Motion of charged particles in homogeneous Kähler and homogeneous Sasakian manifolds, preprint.

Present Address:

Department of Mathematics, Nagoya Institute of TeChnology,

NAGOYA 466-8555, JAPAN.

e-mail: adachi@nitech.ac.jp 